\title{
Effect of elongation on energetic particle-induced geodesic acoustic mode
}

\author{
A. Di Siena, ${ }^{*}$ A. Biancalani, T. Görler, H. Doerk, I. Novikau, \\ P. Lauber, A. Bottino, E. Poli, and the ASDEX Upgrade Team \\ Max Planck Institute for Plasma Physics Boltzmannstr. 285748 Garching Germany
}

(Dated: August 3, 2018)

\begin{abstract}
The effect of the plasma elongation on the energetic-particle-induced geodesic acoustic mode (EGAM) dynamics is studied with numerical simulations performed with the gyrokinetic codes GENE and ORB5 and assessed with analytical models. The former code has been recently extended to support non-Maxwellian distribution functions and for the first time global results are shown here and benchmarked against the code ORB5. Taking advantage of these recent developments in GENE, which allow a joint investigation with ORB5, linear electrostatic simulations with adiabatic electrons have been performed. The impact of the elongation on the EGAM dynamics and excitation mechanism is investigated through the study of the energy exchange terms between the particle and the mode for different plasma geometry. Finally, the results are applied to the study of an ASDEX Upgrade discharge with strongly elongated plasma employing realistic density and temperature profiles.
\end{abstract}

* alessandro.di.siena@ipp.mpg.de 


\section{INTRODUCTION}

The interaction between energetic particles (EP) and plasma turbulence is a central issue for present-day and future magnetic confinement devices, like Tokamaks. In such machines, the substantial plasma temperature, required for thermonuclear reactions to occur, is often produced by energetic ions. In nowadays experiments, they are commonly generated through auxiliary heating systems, such as neutral beam injection (NBI) and ion cyclotron resonance heating (ICRH), and for the case of reactors like ITER, from fusion reactions. In several experimental discharges at AUG[1], JET[2, 3] and Alcator C-Mod[4, 5], a significant stabilisation of the background turbulence and a corresponding steepening of the thermal profiles were observed in the presence of energetic ions. Numerical gyrokinetic simulations, performed on a single magnetic surface, have helped to develop some understanding of these findings, especially through the observation of a strong suppression of ion-scale instabilities like ion-temperature-gradient modes (ITG)[1, 6], mainly attributed to nonlinear electromagnetic effects $[1,6]$. Recently, however, it has been shown that substantial fast ion turbulence stabilisation might be achieved, in particular regimes, even due to electrostatic effects [7]. These numerical observations, corroborated by experimental evidences [8] have increased the interest in electrostatic fast ion physics. However, on the other side, EP may also be deleterious for plasma stability [9-13]. Besides Alfvén type instabilities, they can excite and drive global electrostatic field oscillations, commonly called geodesic acoustic modes (GAM) [14, 15]. These modes are zonal flows $(\mathrm{ZF})[16,17]$ with finite frequency oscillations and are usually damped by collisionless Landau damping in the plasma core or by collisions at the edge of the device [18]. On the other hand, zero frequency zonal flows (ZFZF) [19] are damped by collisional damping only. However, energetic particles might overcome the thermal damping and drive geodesic acoustic modes as stated above, which are called, 
in this case, energetic particle-induced GAM (EGAM) [20-25]. The parallel velocity anisotropies arising from the highly non-thermalised fast ion distribution function may indeed lead to an energy transfer from the energetic ions to the EGAM via inverse Landau damping and to a growth of this mode. The presence of energeticparticle-induced geodesic acoustic modes, excited through external NBI and ICRH heating, has also been observed experimentally in different plasma devices and in different plasma conditions at DIII-D [26], LHD [27] ASDEX Upgrade [28] and JET [11]. Furthermore, Ref. [29] contains some first evidence for EGAM excitation by energetic electrons in experiments at HL-2A. The different fast ion effects on plasma turbulence are strongly nonlinearly connected. Indeed, the macroscopic ion-scale structures, generated by ITG turbulence and the radial ZF oscillations, generated via Reynold stress are nonlinearly coupled. Furthermore, ITG turbulence mainly saturates via ZFs and any modification of the latter may therefore have a strong impact on transport levels and hence on energy confinement. Hereafter, EGAMs could be another interesting player in this context. Although significant improvement in the theoretical understanding of the EGAM linear and nonlinear dynamics has already been achieved recently [30-32], several aspects still need to be addressed in order to be able to understand the whole complex turbulence/ZF nonlinear interaction. The aim of the present work is to analyse the impact of realistic magnetic configurations on the linear electrostatic EGAM physics. More specifically, the effect of the elongation on the EGAM linear dynamics is studied with the gyrokinetic codes GENE [33] and ORB5 [34, 35]. The former has been recently extended to support arbitrary non-Maxwellian fast ion backgrounds and in the contribution at hand, results obtained studying the whole radial domain (global simulations) are presented for the first time. In Sec. II, a brief description of the gyrokinetic codes GENE and ORB5 and of the distribution functions employed in this work is provided. In the framework of verification of the newly implemented terms, the GENE results are 
benchmarked with the gyrokinetic code ORB5 both in circular and realistic geometries, respectively in Sec. III and Sec. IV. A theoretical and numerical interpretation of the results obtained in different realistic plasma configurations is presented in Sec. V and Sec. VI with the help of simplified analytical theory. In the last section of this paper, namely Sec. VII, the study of a realistic ASDEX Upgrade experimental scenario with a strongly elongated plasma is investigated employing realistic density and temperature profiles.

\section{DESCRIPTION OF THE GYROKINETIC CODES GENE AND ORB5}

\section{A. The GENE code}

The Gyrokinetic Electromagnetic Numerical Experiment (GENE) solves numerically the Vlasov-Maxwell system of equations for each time step on the five dimensional grid $\left(\vec{R}, v_{\|}, \mu\right)$, where $\vec{R}$ represents the gyrocenter position, $v_{\|}$the velocity component parallel to the magnetic field and $\mu=m_{s} v_{\perp}^{2} /\left(2 B_{0}\right)$ the magnetic moment. Here $m_{s}$ denotes the mass of species $s, v_{\perp}$ the velocity component perpendicular to the magnetic field and $B_{0}$ the background magnetic field. GENE can either be operated in a flux tube simulation domain (employing a local approximation) [33], in a radially global torus geometry [36], or as a flux-surface code [37]. Furthermore, full electromagnetic effects, a realistic collision operator and experimental geometries can be included. The gyrokinetic representation of each species is done employing the so-called $\delta f$ approach. The distribution function is split into a background component and in a small fluctuating part, i.e. $F_{s}=F_{0, s}+f_{1, s} \cdot F_{0, s}$ is usually assumed to be a local Maxwellian distribution with $\partial F_{0} / \partial t=0$, defined - in normalised units 
- as follows

$$
F_{0, s}=\frac{\hat{n}_{s}}{\left(\pi \hat{T}_{s}\right)^{3 / 2}} e^{-\frac{v_{11}^{2}+\mu B_{0}}{\hat{T}_{s}}} .
$$

Here, the velocities are normalised to the thermal velocity of each species $(\mathrm{s}) v_{s, t h}=$ $\sqrt{2 T_{s}\left(x_{0}\right) / m_{s}}$ - in units of $c_{s}=\sqrt{T_{e}\left(x_{0}\right) / m_{i}}$ and the following abbreviations have been introduced for density and temperature profiles $\hat{n}_{s}=n_{s}(x) / n_{e}\left(x_{0}\right)$ and $\hat{T}_{s}=$ $T_{s}(x) / T_{e}\left(x_{0}\right)$ with respect to the reference position $x_{0}$. In GENE the reference values are the main ion mass $m_{i}$, electron temperature $T_{e}$ and a reference magnetic field $B_{\text {ref }}$, see Ref. 36 for more details. Furthermore, recently, GENE has been extended in order to run and support arbitrary background distributions [38], which may be either analytical or numerical [39]. These new capabilities allow to highly increase the realism in the modelling tools of the non-thermalised energetic ion population. In Ref. 39 both the analytical framework and numerical results were shown employing the extended version of the code on a single magnetic surface, i.e. flux-tube simulations. In the contribution at hand, results obtained studying the whole radial domain are presented for the first time and benchmarked with the gyrokinetic global code ORB5 $[34,35]$. We limit our investigation to the Geodesic Acoustic Modes (GAM) physics in electrostatic plasmas. GAMs are axisymmetric modes $(n=0)$ with poloidally symmetric potential $(m=0)$ and asymmetric density $(m=1)$ perturbations, which are usually suppressed through Landau damping mechanisms. However, in the presence of energetic ions, unstable (growing) modes can be excited by velocity space gradients in the non-thermalised fast ion distribution. In Sec. III,IV,VII, analogously as in Ref. 23, 30, and 31, the fast ion background is assumed to be a (symmetric double) bump-on-tail distribution defined - in normalised units - as follows

$$
F_{E P}=\frac{\hat{n}_{E P}}{\left(\pi \hat{T}_{E P}\right)^{3 / 2}} e^{-\frac{v_{\|}^{2}+\mu B_{0}}{\hat{T}_{E P}}} e^{-\frac{\bar{v}_{\|}^{2}}{\hat{T}_{E P}}} \cosh \left(\frac{2 v_{\|} \bar{v}_{\|}}{\hat{T}_{E P}}\right)
$$


Similar to Refs. 23, 30, and 31 the slow time dependence of the energetic particle background is considered irrelevant on the EGAM linear time scales both in ORB5 and GENE.

\section{B. The ORB5 code}

ORB5 is a Lagrangian electromagnetic particle-in-cell (PIC) gyrokinetic code, which operates on the full global radial domain. It solves numerically the VlasovMaxwell equations in a five dimensional space $\left(\vec{R}, p_{\|}, \mu\right)$, where $\vec{R}$ denotes the gyrocenter position, $p_{\|}=m_{s} v_{\|}+\left(q_{s} / c\right) \tilde{A}_{\|}$the canonical parallel momentum and $\mu=$ $m_{s} v_{\perp}^{2} /\left(2 B_{0}\right)$ the magnetic moment of the species s. Here, $q_{s}$ represents the s-species charge, $c$ the speed of light and $\tilde{A}_{\|}$the gyroaveraged parallel component of the vector potential. Similarly to GENE, ORB5 splits the distribution function in a background component and in a fluctuating part. However, ORB5 discretises the distribution functions with particles, so called markers, and weights. The system evolves in time by pushing the particle markers along the trajectories derived from the gyrokinetic model and the fields are evaluated by solving the gyrokinetic field equations, properly projecting the marker weights on a spatial grid. ORB5 has the possibility to initialise the fast ion background distribution function both as an equivalent Maxwellian background, as defined in Eq. (1) and with a double symmetric bumpon-tail distribution, as defined in Eq. (2). Furthermore, the velocities of each species are normalised to the thermal velocity of the main ion (i) defined as $v_{i, t h}=\sqrt{T_{i} / m_{i}}$. With the aforementioned normalisation, a factor of $\sqrt{2}$ of difference compared to the GENE normalisation appears for the cases of $T_{E P}=T_{i}$ and a factor of $\sqrt{2} T_{E P} / T_{i}$ for realistic fast ion temperatures. Further details on the ORB5 basic equations and on the code implementation can be found in Ref. 34 and 35. 


\section{NUMERICAL RESULTS WITH CIRCULAR GEOMETRY}

Linear electrostatic benchmarks between GENE and ORB5 are performed studying the EGAM growth rates and frequencies for different fast particle concentrations and different magnetic circular geometries. This first numerical analysis allows one to verify the newly extended global version of the gyrokinetic code GENE, which includes non-Maxwellian distribution functions for the energetic ion population as described in the previous section. Electrons are considered adiabatic and at $t=0 \mathrm{a}$ density perturbation of the form $n_{1}\left(\rho_{\text {pol }}, t_{0}\right) \sim \sin \left(\pi \rho_{\text {pol }}\right)$ is initialised and Dirichlet boundary conditions are applied. Here, $\rho_{p o l}=\sqrt{\Psi_{p} / \Psi_{p, e d g e}}$ represents the radial coordinate with values in $[0,1]$ and $\Psi_{p}$ the poloidal flux. The growth rates and frequencies are obtained through a logarithmic fit of the time evolution of $\phi_{1}$ at the radial position $\rho_{\text {pol }}=0.5$. The EGAMs can be excited only through an energy transfer from the fast particle population and the mode. In order for this mechanism to occur an inverse Landau damping is required, i.e. a non-Maxwellian distribution function must be used for modelling the fast particle population. To this aim, both GENE and ORB5 consider the bump-on-tail distribution function defined in Eq. (2) while a local Maxwellian is maintained for the main (thermal) ion species.

\section{A. Simulation results}

All the numerical simulations presented in this section have been performed with analytical circular magnetic equilibria [40] and in a collisionless plasma. The inverse aspect ratio $\epsilon\left(=a / R_{0}\right)$ is fixed to 0.3125 , with $R_{0}=1 \mathrm{~m}$ and $a=0.3125 \mathrm{~m}$. The magnetic field on axis is equal to $B_{0}=1.9 \mathrm{~T}$. Furthermore, flat temperature and density profiles have been considered throughout all the rest of this section with the radial coordinate which goes from $0<\rho_{\text {pol }}<1$. The EP and bulk ion temperatures 

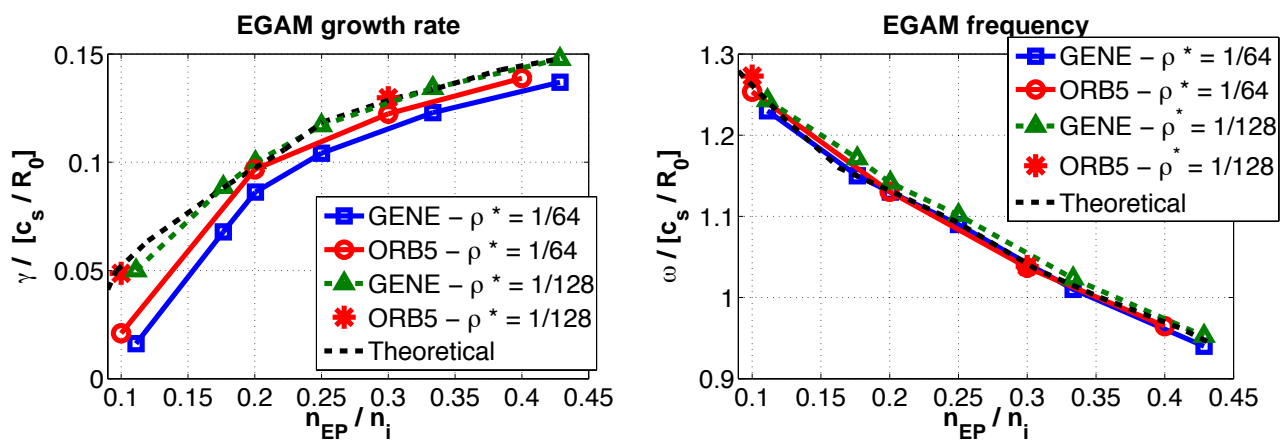

FIG. 1: EGAM frequencies and growth rates for $q=3$ and $\rho^{*}=1 / 64 ; \rho^{*}=1 / 128$.

$\left(T_{e}=T_{i}=T_{E P}\right)$ are fixed by the choice of $\rho^{*}=\rho_{s} / a$, with $\rho_{s}=\sqrt{T_{e} / m_{i}} / \Omega_{i}$ and $\Omega_{i}=q B_{0} / m c$. In the following two different values of $\rho^{*}$ have been used, i.e. $\rho^{*}=1 / 64$ and $\rho^{*}=1 / 128$. The fast ion temperature and the values of $\rho^{*}$ have been chosen to reduce the computation effort of the numerical simulations. The reference density, on the other hand, is a free parameter in collisionless electrostatic simulations as none of the physics inputs is constrained by this value and the chosen linear observables do not depend on the density either. Furthermore, the value of $\bar{v}_{\|}$, which determines the magnitude of the shift in $v_{\|}$, has been fixed to 2.83 in units of ion thermal velocity (=4 in ORB5 units). In Fig. (1) EGAM growth rates and frequencies obtained for different energetic ion concentrations at $\rho_{\text {pol }}=0.5$ are compared between GENE and ORB5 for a flat q-profile $=3$, i.e. shear $=0$ and for $\rho^{*}=1 / 64$ and $\rho^{*}=1 / 128$. The theoretical values of the EGAM frequencies and growth rates have been taken from Ref. 31. The differences between the GENE and ORB5 results are as the order of $7 \%$ for $\rho^{*}=1 / 64$, which reduce to $\sim 5 \%$ for $\rho^{*}$, i.e. $\rho^{*}=1 / 128$. The agreement between the codes improves even further if the value of the flat q-profile is reduced to 2, as it is shown in Fig. (2) for the EGAM growth rates and frequencies. For this specific geometry, i.e. $q=2$, and for the fast ion 

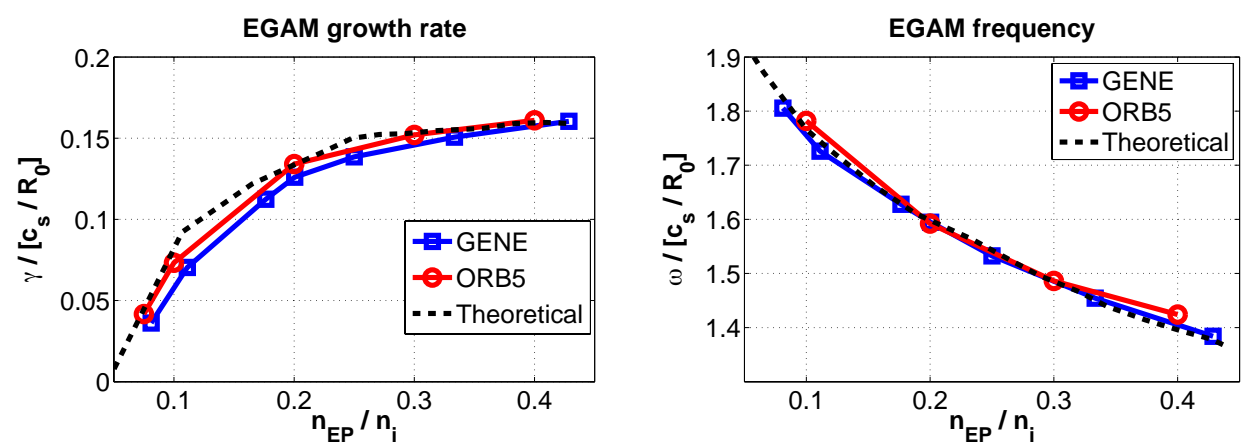

FIG. 2: EGAM frequencies and growth rates for $q=2$ and $\rho^{*}=1 / 64$.

concentration of $n_{E P} / n_{e}=0.2$, a comparison of the radial structure of the perturbed electrostatic potential obtained with GENE and ORB5 is shown in Fig. (3) for $t=0$ and $t=268.1$ in units of $c_{s} / R_{0}$. The time $t=268.1 c_{s} / R_{0}$ corresponds to a maximum of the electrostatic potential. This time has been chosen after an initial transient phase, and therefore gives a characteristic indication of the radial structure of the well-formed mode. A good agreement between the codes is observed. In particular, a slight reduction of the radial wave number for the formed mode, with respect to the value of the initialized field, is found, and a slight reduction of the radial position of the peak. The latter corresponds to a lack of radial symmetry caused by the dependence of the aspect ratio on the radius.

\section{NUMERICAL RESULTS IN ELONGATED GEOMETRY}

In the following section, the newly extended version of the gyrokinetic code GENE is employed for a joint investigation with ORB5 of the effect of the elongation on the EGAM dynamics. An initial denisty perturbation is excited at $t=0$ and its evolution in time with linear collisionless electrostatic simulations with flat profiles is observed. The growth rates and frequencies are obtained through a logarithmic 


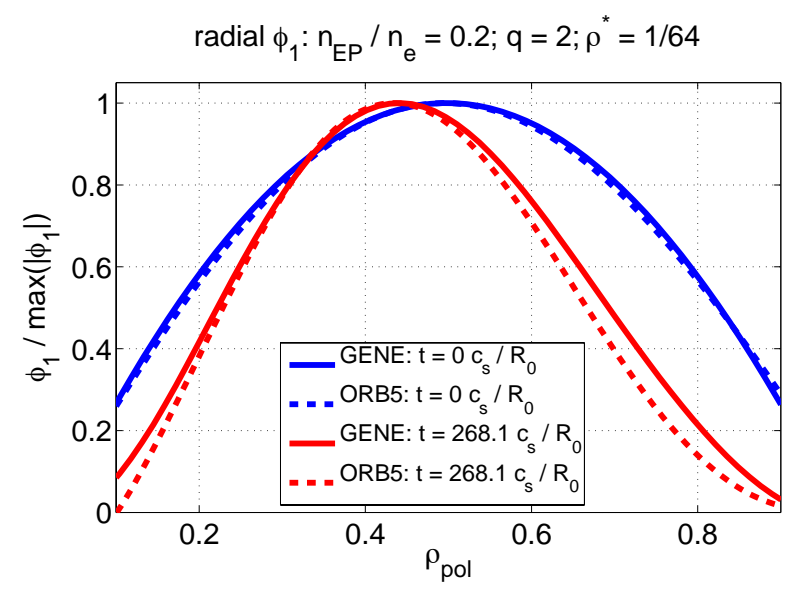

FIG. 3: Comparison of the radial structure of the electrostatic potential $\phi_{1}$ between GENE (continuous line) and ORB5 (dotted line) for the case $n_{E P} / n_{e}=0.2, q=2$ and $\rho^{*}=1 / 64$.

fit of the time evolution of $\phi_{1}$ at the radial position $\rho_{p o l}=0.5$. The choice of using a reduced setup, neglecting radial profiles and magnetic fluctuations, is motivated by the need of decreasing the physical complexity of the system.

\section{A. Simulation setup and results}

All the numerical simulations presented in this section have been performed with numerical CHEASE equilibria [41]. The physical parameters are the same as the ones in the previous section and the value of $\rho^{*}$ is fixed to $\rho^{*}=\rho_{s} / a=1 / 128$. The q-profile is almost constant at the value of $q=2$ in the whole range of the radial profile which goes from $0<\rho_{\text {pol }}<1$ and the elongation is varied from $\kappa=1$ (circular flux surfaces - see Fig. (4) a) -) to $\kappa=1.75$ (elongated plasma - see Fig. (4) b) -). At $t=0$ the same sinusoidal density perturbation defined in the previous section is initialised and allowed to evolve in time enforcing Dirichlet boundary conditions. 

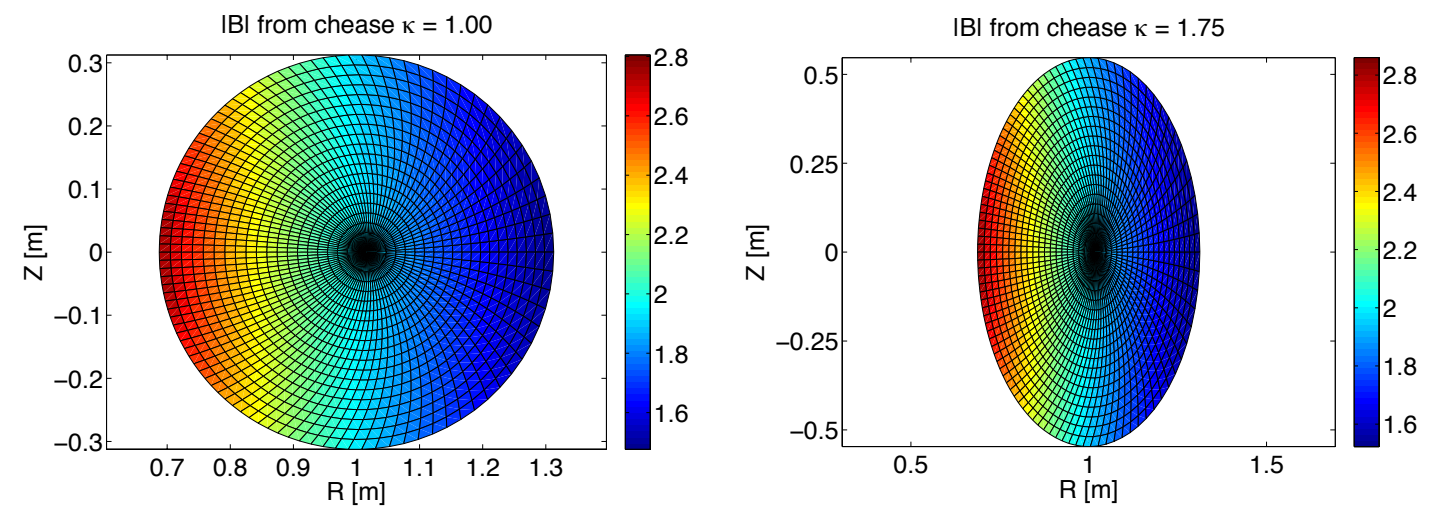

FIG. 4: Intensity of the equilibrium magnetic field on the flux-coordinate grid for

a) $\kappa=1.00$ and b) $\kappa=1.75$.
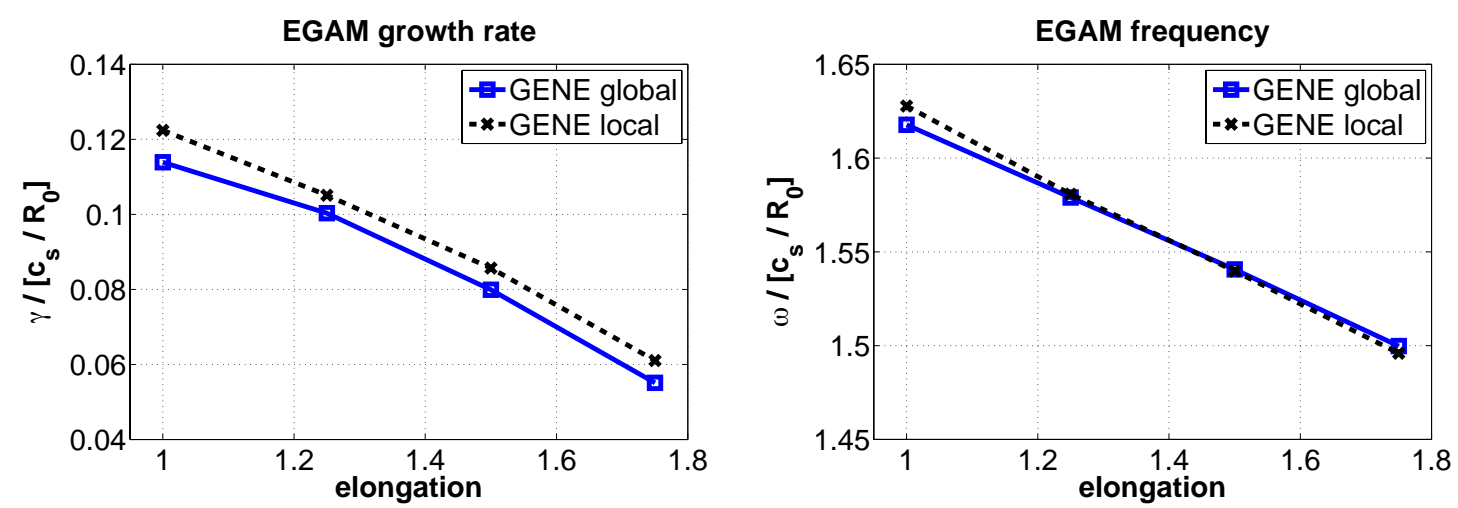

FIG. 5: EGAM frequencies and growth rates for different values of the elongation and for $n_{E P} / n_{e}=0.15$ at $\rho^{*}=1 / 128$ for $q=2$.

In Fig. (5)-(6), linear EGAM growth rates and frequencies are shown for different values of the elongation and for different energetic ion concentrations. The latter is defined as the energetic particle density divided by the electron density. From Fig. (6) it is possible to observe the excellent agreement between the codes GENE 

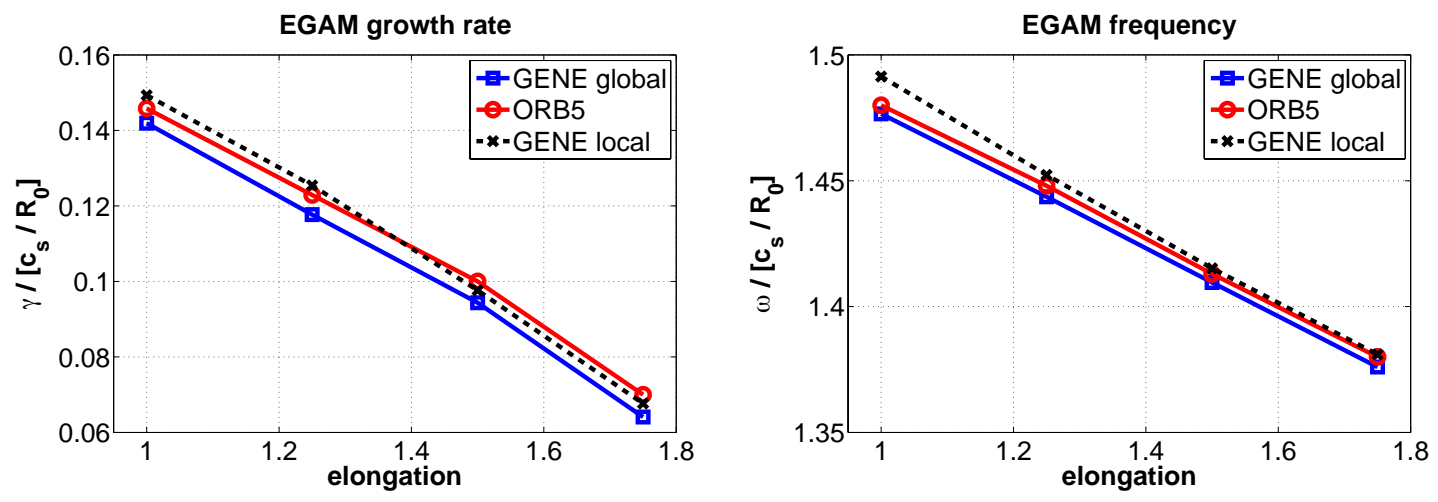

FIG. 6: EGAM frequencies and growth rates for different values of the elongation and for $n_{E P} / n_{e}=0.23$ at $\rho^{*}=1 / 128$ for $\mathrm{q}=2$.
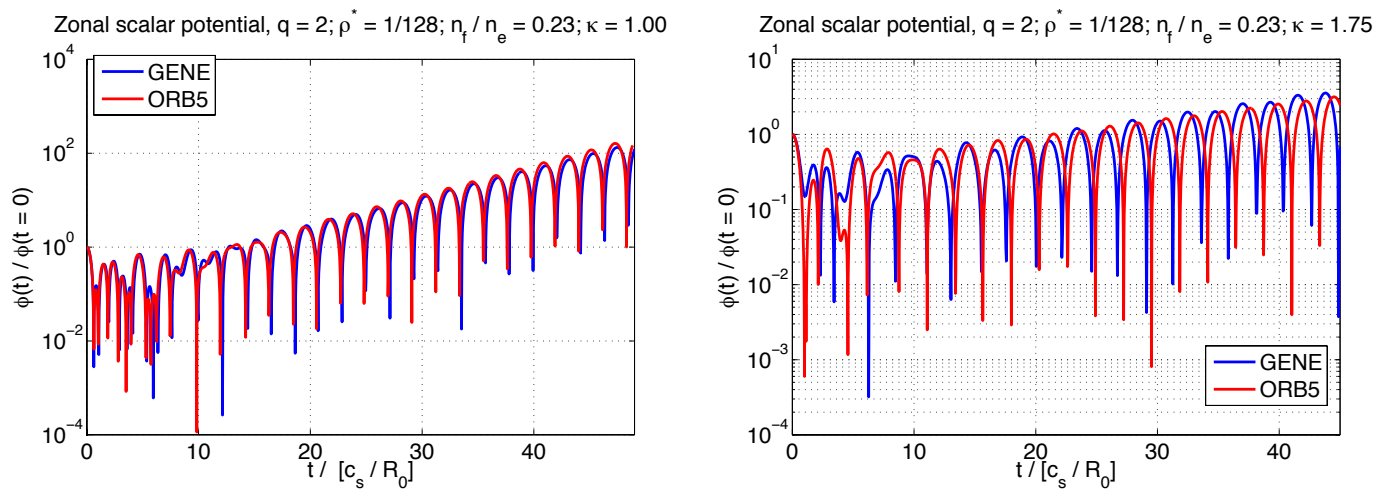

FIG. 7: Comparison between the scalar potential normalised at $\phi(t=0)$ obtained from GENE (blue line) and ORB5 (red line) for $n_{E P} / n_{e}=0.23$ at $\rho^{*}=1 / 128$ for $\mathrm{q}$

$$
\text { =2: a) } \kappa=1.00 \text { and b) } \kappa=1.75
$$

and ORB5 for the fast ion concentrations of $n_{E P} / n_{e}=0.23$. This agreement is also confirmed by the study of the time trace of the perturbed potential $\phi_{1}$ for different values of the plasma elongation, as can be seen in Fig. (7). Furthermore, we observe a pronounced dependence of the EGAM growth rate - which decreases, i.e. is less 
destabilised - with the elongation and only weak changes in the mode frequency.

\section{REDUCED GYROKINETIC MODEL FOR THE FAST ION ENERGY TRANSFER TERMS}

The theoretical framework used for the physical interpretation of the numerical results of the effect of the plasma elongation on the EGAM dynamics is here presented, following the analysis of Ref. 42 and 43. The perturbed part of the linear electrostatic Vlasov equation in GENE normalised units can be written, without doing any assumption of the background distribution function and neglecting the trapped fast ion term, as follows

$$
\begin{aligned}
\frac{\partial f_{1, s}}{\partial t}= & -\frac{\mathcal{C}}{\mathcal{J} B_{0}} v_{\|} v_{t h}\left[\frac{\partial f_{1, s}}{\partial \theta}-\frac{q_{s}}{2 T_{0, s} v_{\|}} \partial_{\theta} \bar{\phi}_{1} \frac{\partial F_{0, s}}{\partial v_{\|}}\right] \\
& -\frac{T_{0, s}}{q_{s}} \frac{\left(\mu B_{0}+2 v_{\|}^{2}\right)}{B_{0}} \mathcal{K}_{x} \frac{\partial f_{1, s}}{\partial x}+\frac{1}{2 v_{\|}} \frac{\left(\mu B_{0}+2 v_{\|}^{2}\right)}{B_{0}} \mathcal{K}_{x} \frac{\partial \bar{\phi}_{1}}{\partial x} .
\end{aligned}
$$

Here, we have defined the following geometrical coefficients $\mathcal{K}_{x}=-\left(\left(\mathbf{B}_{0} \times \nabla B_{0}\right)\right.$. $\hat{x}) / B_{0}^{2}, \mathcal{C}^{2}=\mathbf{B}_{0} \cdot \mathbf{B}_{0}$ and $\mathcal{J}^{-1}=\mathbf{B}_{0} \cdot \nabla \theta / \mathcal{C}$ in the field aligned coordinate system with

$x$ radial direction, $\theta$ field aligned direction and $z$ binormal direction. Furthermore, $\bar{\phi}_{1}$ denotes the gyroaveraged potential. The reference values used for normalizing Eq. 3 are the elementary electron charge $e$, the main ion mass $m_{i}$, electron temperature $T_{e}$, a reference magnetic field $B_{\text {ref }}$ and a macroscopic length $L_{\text {ref }}$, see Ref. 36 for more details. The frequencies have been normalised to $c_{s} / R_{0}$. It is possible to perform the following Fourier and Laplace transformation with respect to $k_{x}, \theta$ and $t$ for the perturbed quantities

$$
f_{1, s}(\mathbf{x}, \theta, t)=\sum_{m}(2 \pi)^{-1} \int d k_{x} d \omega f_{1, s}\left(k_{x}, \omega\right) e^{i\left(m \theta-\omega t+k_{x} x_{s}\right)},
$$


where, $x_{s}=\delta_{s} \cos \theta, \delta_{s}=\frac{\epsilon v_{t h, s}}{v_{\|}}\left(v_{\|}^{2}+B_{0} \mu / 2\right)$ and $\epsilon=a / R_{0}$. Positive values of the real frequency identify a mode propagating in the ion direction. Employing the JacobiAnger expansion, respectively, $e^{i k_{x} \delta_{s} \cos \theta}=\sum_{l} i^{l} J_{l}\left(k_{x} \delta_{s}\right) e^{i l \theta}$ and Eq. (4), Eq. (3) can be re-written as follows

$$
f_{1, m, s}=\sum_{l, l^{\prime}}-\frac{q_{s}}{2 T_{0, s} v_{\|}} \frac{\partial F_{0, s}}{\partial v_{\|}} i^{\left(l^{\prime}-l\right)} J_{l^{\prime}}\left(k_{x} \delta_{s}\right) J_{l}\left(k_{x} \delta_{s}\right) \frac{\left[\omega_{d s}+(l+m) \omega_{t}\right]}{\left[\omega-\omega_{t}(l+m)+\omega_{d s}\right]} \phi_{1, m+l-l^{\prime}},
$$

where, $\omega_{d s}=\frac{T_{0, s}}{q B_{0}}\left(\mu B_{0}+2 v_{\|}^{2}\right) \mathcal{K}_{x} k_{x}$ is the toroidal drift frequency and $\omega_{t}=\frac{\mathcal{C}}{q B_{0} \mathcal{J}} v_{\|} v_{t h}$ is the transit frequency. Furthermore, the orthogonality relation yields $l+m=l^{\prime}+m^{\prime}$. For an $s-\alpha$ magnetic equilibrium, the transit frequency can be rewritten as $\omega_{t}=$ $\frac{v_{11} v_{t h}}{q}$, where $q$ represents the safety factor. Since $k_{x, \min }=2 \pi \rho^{*} \ll 1$, it is possible to neglect the toroidal drift frequency $\omega_{d s}$ with respect to the transit frequency $\omega_{t}$ and re-write Eq. (5) as

$$
f_{1, m, s}=\sum_{l, l^{\prime}}-\frac{q_{s}}{2 T_{0, s} v_{\|}} \frac{\partial F_{0, s}}{\partial v_{\|}} i^{\left(l^{\prime}-l\right)} J_{l^{\prime}}\left(k_{x} \delta_{s}\right) J_{l}\left(k_{x} \delta_{s}\right) \frac{(l+m) \omega_{t}}{\left[\omega-\omega_{t}(l+m)\right]} \phi_{1, m+l-l^{\prime}}
$$

Up to the first order in $m^{\prime}$ it is possible to write

$$
f_{1, m=1, s}=-\frac{q_{s} J_{0}^{2}\left(k_{x} \delta_{s}\right)}{2 T_{0, s} v_{\|}} \frac{\partial F_{0, s}}{\partial v_{\|}} \frac{\omega_{t} \phi_{1, m^{\prime}=1}}{\omega-\omega_{t}}-\frac{i q_{s} J_{1}\left(k_{x} \delta_{s}\right) J_{0}\left(k_{x} \delta_{s}\right)}{2 T_{0, s} v_{\|}} \frac{\partial F_{0, s}}{\partial v_{\|}} \frac{\omega_{t} \phi_{1, m^{\prime}=0}}{\omega-\omega_{t}} .
$$

The higher order resonance terms are here neglected. The GAM dispersion relation can be obtained from Eq. (6) by solving the Poisson equation. For the case of $s-\alpha$ geometry, adiabatic electrons and equivalent Maxwellian distributed particles the dispersion relation of Ref. 42 is obtained. If a fraction of fast ions, modelled with the bump-on-tail distribution of Eq. (2) is considered, the dispersion relation of Ref. 24 and 31 is instead derived from Eq. (6). The reduced Vlasov equation derived in Eq. (7) can be used to investigate the contribution of each particle species $(s)$ to the overall energy exchange between the particles and the mode through the energy 
conservation property of the Vlasov-Poisson equations [44, 45]. This analysis allows one to study and separate any fast-ion drive mechanisms from the thermal damping of the bulk plasma and will be used in the next section to identify the resonance position for each value of the plasma elongation. Thereafter, the energy exchange between the particles and the mode can be written as

$$
\frac{\partial E_{s}}{\partial t}=\mathbb{R} e\left\{\int \pi B_{0}\left(v_{\|}^{2}+\mu B_{0}\right) \frac{\partial f_{1, s}}{\partial t} d^{3} x d v_{\Perp} d \mu\right\},
$$

where $f_{1, s}$ denotes the perturbed distribution function of the species $s$. From Eq. (8) it is possible to study the contribution of each term of the Vlasov equation to the energy exchange and hence to the mode excitation. Positives (negatives) values of $\frac{\partial E_{s}}{\partial t}$ indicate that the plasma species considered is giving (taking) energy to (from) the electrostatic field component with a consequent growth (damping) of the mode. As it is shown in Ref. 44, Eq. (8) can be written as the sum of curvature and parallel term contributions. In Fig. (8) the contribution of each of these terms to the total energy derivative is displayed (a) for the thermal and (b) for the energetic ions at $n_{E P} / n_{e}=$ $0.15 ; q=2 ; \rho^{*}=1 / 128 ; \kappa=1.00$. Apparently, the main contributions stem from the curvature, which is consistent with fast ions exciting a geodesic acoustic mode and the parallel advection terms, which although overall smaller, can become significant at the minimum of the curvature term - these two different contributions oscillate, indeed, with opposite phase relation. This picture does not depend on the elongation and it is qualitative the same between thermal and fast ions. Furthermore, we observe that the fast particles, modelled with the bump-on-tail distribution defined in Section II, act as a positive drive to the EGAM with a positive contribution to the particles/mode power exchange. The thermal ions, on the contrary, globally damp the fast ion driven mode, even if locally they can still provide energy [31]. The overall contribution (drive-damping) to the EGAM mode determines the total mode growth rate. 

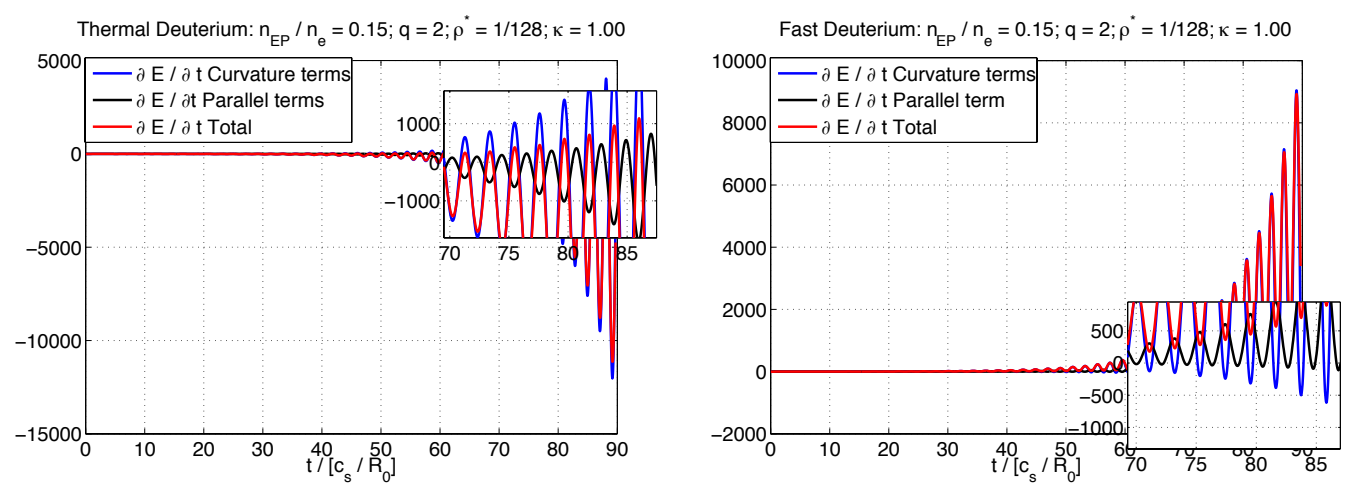

FIG. 8: Study of the different contributions of the thermal and fast ions to the total energy transfer.

\section{THEORETICAL MODEL}

The effect of the plasma elongation on the resonance positions for both thermal and energetic ions are studied in this section with gyrokinetic simulations and the results compared to analytical predictions. For the small orbit drift width limit, the dominant resonance mechanism is predicted to occur between the GAM linear mode frequency and the bulk-ion parallel motion, described by the transit frequency $\omega_{t}$ [46]. The latter, averaged over the poloidal angle, is defined in GENE normalised units as follow [46]

$$
\omega_{t}=\frac{v_{\|} v_{t h, i}}{q}\left[1+\frac{\epsilon^{2}}{2}-\frac{\epsilon^{2}\left(\kappa^{2}+1\right)}{4 q^{2}}\right] .
$$

Here $\kappa$ represents the value of the plasma elongation. In Fig. (9) a qualitatively good agreement (inside error bars) between GENE/ORB5 and the theoretical predictions of Ref. 46 is shown for the GAM damping rate and frequency for different values of the elongation. The relatively large error bars of Fig. (9)(a) are a consequence of the significantly smaller GAM damping rate, which makes the corresponding analysis more challenging. The geometry and the plasma parameters are the same of the ones 

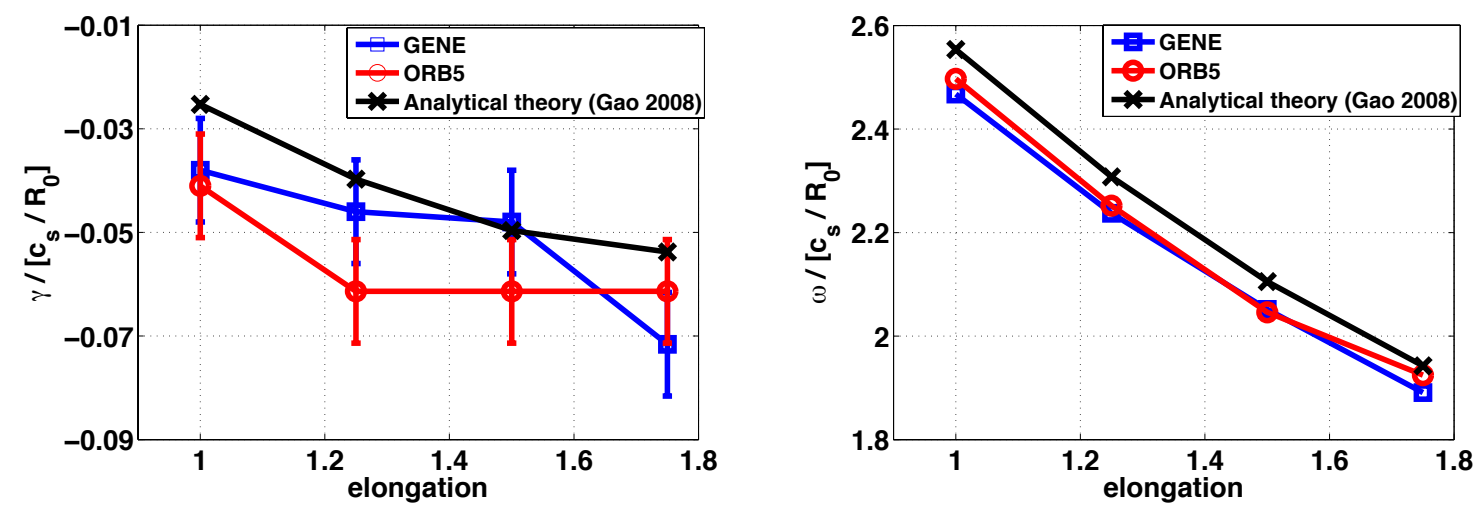

FIG. 9: Comparison between the theoretical prediction of Ref. 46 and the GENE (blue line)/ORB5 (red line) GAM damping rate a) and frequency b) for different values of the elongation for the case $q=2$ and $\rho^{*}=1 / 128$.

employed in the previous section, i.e. $\rho^{*}=1 / 128$ and $q=2$. The mild dependence of the GAM damping rate with the elongation can be explained studying the $v_{\|}$ structure of the energy exchange term. Inserting Eq. (6) in Eq. (8) yields the following simplified expression for the energy exchange power between the particles and the mode, i.e. the energy taken (negative) or given (positive) from/to the mode

$$
\begin{aligned}
\frac{\partial E_{s}}{\partial t}= & \mathbb{R} e\left\{-\int i \omega \pi B_{0}\left(v_{\|}^{2}+\mu B_{0}\right) f_{1, m=1} d^{3} x d v_{\|} d \mu\right\} \\
= & \mathbb{R} e\left\{\int i \omega \pi B _ { 0 } ( v _ { \| } ^ { 2 } + \mu B _ { 0 } ) \left[\frac{q_{s} J_{0}^{2}\left(k_{x} \delta_{s}\right)}{2 T_{0, s} v_{\|}} \frac{\partial F_{0, s}}{\partial v_{\|}} \frac{\omega_{t} \phi_{1, m^{\prime}=1}}{\omega-\omega_{t}}\right.\right. \\
& \left.\left.\quad \frac{i q_{s} J_{1}\left(k_{x} \delta_{s}\right) J_{0}\left(k_{x} \delta_{s}\right)}{2 T_{0, s} v_{\|}} \frac{\partial F_{0, s}}{\partial v_{\|}} \frac{\omega_{t} \phi_{1, m^{\prime}=0}}{\omega-\omega_{t}}\right] d^{3} x d v_{\|} d \mu\right\} .
\end{aligned}
$$

This relation is consistent with the ones obtained in Ref. 23 and demonstrates that the energy transfer between the GAM/EGAM and the plasma species is proportional to the $v_{\|}$derivative of the background distribution function, i.e. $\frac{\partial F_{0}}{\partial v_{\|}}$. If the plasma contains only thermal ions, i.e. can be described by a Maxwellian background, the 

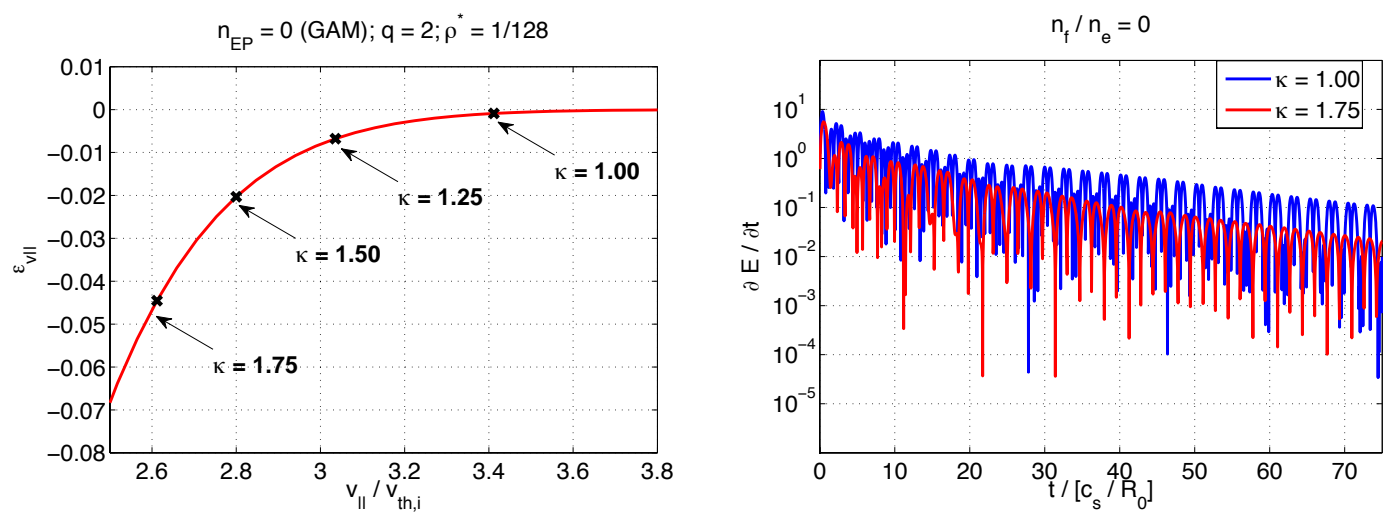

FIG. 10: Case without fast ions: a) Slice at $\mu=0$ of $\epsilon_{v_{11}}$ for different values of $v_{\|}$.

The symbols in the figure identify the value of $\epsilon_{v_{1}}$ at the different resonance positions identified with Eq. (9) for each value of the elongation. b) Time evolution of the system energy exchange for $\kappa=1.00$ and $\kappa=1.75$.

exchange power density between the wave and the ions is always negative and the mode is always naturally damped. The magnitude of the damping depends on the value of $\frac{\partial F_{0}}{\partial v_{11}}$ at the parallel transit motion resonance position. Larger values (in magnitude) of $\frac{\partial F_{0}}{\partial v_{\|}}$correspond to larger energy exchange rates and in the case of negative $v_{\|}$derivatives to stronger damping. A $\mu=0$ and $\rho_{\text {pol }}=0.5$ slice of the $v_{\|}$dependent energy exchange term $\epsilon_{v_{\|}}=v_{\|} \omega_{t} \frac{\partial F_{0}}{\partial v_{\|}}$is shown in Fig. (10)a for different values of $v_{\|}$. The symbols in the figure mark the different resonance positions as defined by Eq. (9) for each elongation value. Note that for the case of energetic ions the thermal velocity in Eq. (9) refers to the energetic ion species. With increasing elongation, the resonant parallel velocity moves to smaller values, i.e. for the case of Maxwellian backgrounds to more negative values of $\epsilon_{v_{\|}}$and thus to stronger damping. These findings are consistent with the elongation dependence of the total energy exchange, see Fig. (10)b. However, if non-thermalised fast ions - here modelled with the bump-on-tail distri- 

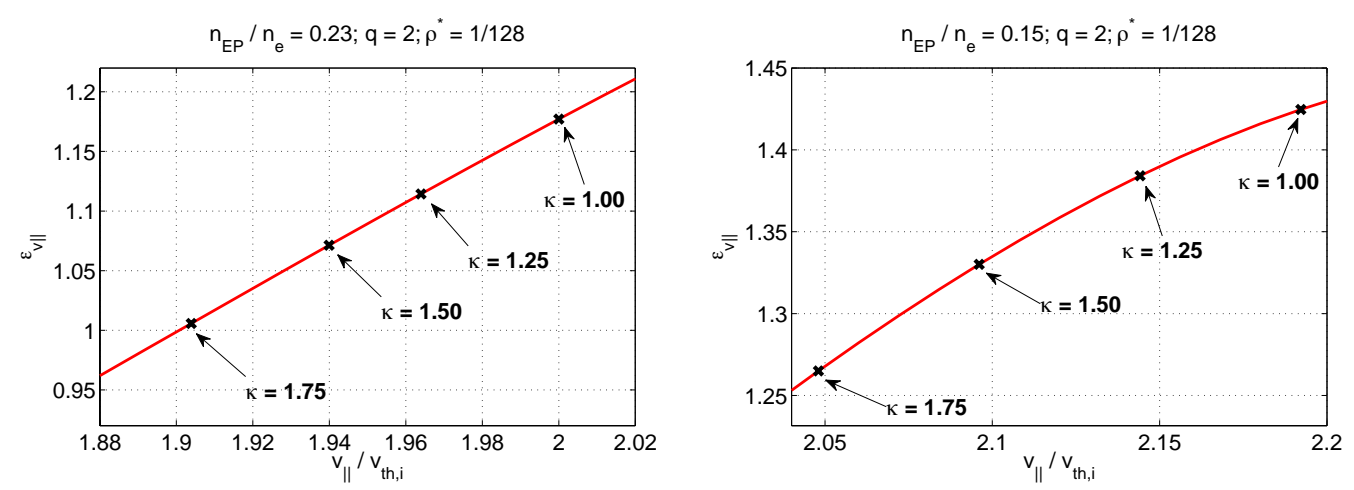

FIG. 11: Slice at $\mu=0$ of $\epsilon_{v_{\|}}$for different values of $v_{\|}$for a) $n_{f} / n_{e}=0.23$ and b) $n_{f} / n_{e}=0.15$. The symbols in the figure mark the values of $\epsilon_{v_{\|}}$at the resonance positions associated to different elongation values, see Eq. (9).

bution defined in Eq. (2) - are considered, the velocity derivative of the background distribution function assumes positive values. A net energy transfer therefore occurs from the fast ions to the mode, which, if the drive overcome the thermal damping, might now be driven by the energetic particles (EGAM) with positive values of $\gamma$. Furthermore, the EGAM growth rate is weakened with increased elongation which resembles the stronger damping in the GAM case above. This change in the $\gamma$ dependence with the elongation is again consistent with the corresponding change in the shape of the velocity space structure of $\epsilon_{v_{\|}}$for a bump-on-tail distribution compared to a Maxwellian background. Indeed, when the resonant transit velocity decreases with the elongation, $\epsilon_{v_{\|}}$also decreases accordingly with a consequent weakening of the wave-fast ion energy transfer. As before, this elongation dependence can also be observed in total energy exchange, see Fig. (12)a-b. Further analysis can be performed studying the velocity space structure of $\partial E / \partial t$, i.e. the energy exchange, for both different elongations, here $\kappa=1.00$ and $\kappa=1.75$, and energetic particle densities, $n_{E P} / n_{e}=0.23$ and $n_{E P} / n_{e}=0.15$. In Fig. (13-14) a slice at $\mu=0$ and 

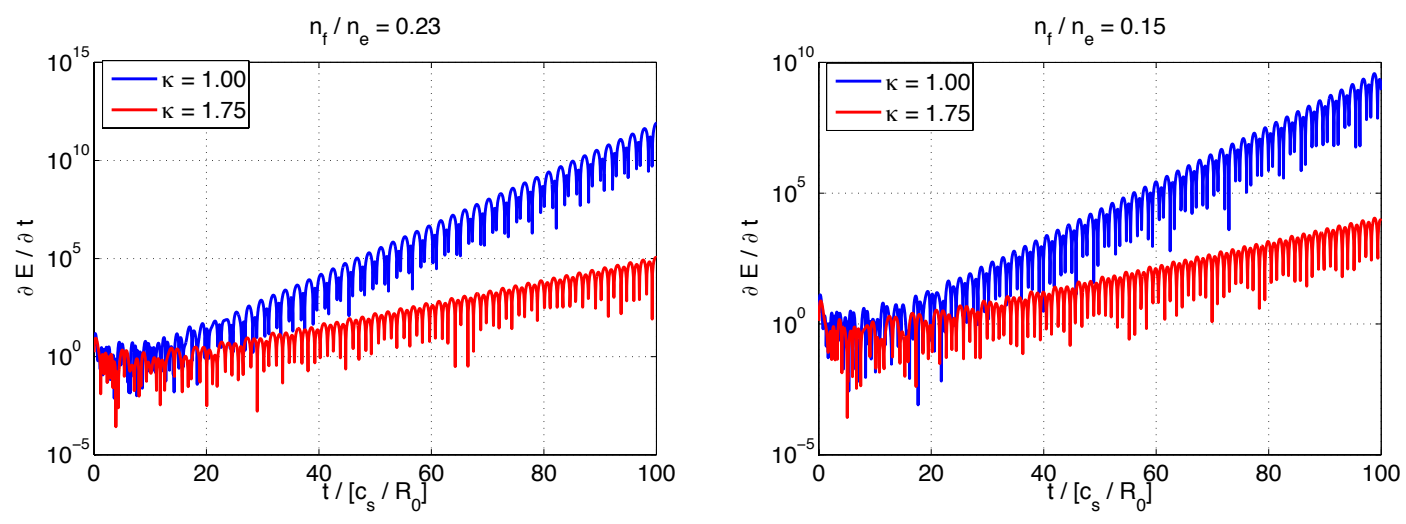

FIG. 12: Time evolution of the total energy exchange for $\kappa=1.00$ and $\kappa=1.75$ for a) $n_{f} / n_{e}=0.23$ and b) $n_{f} / n_{e}=0.15$.

$\rho_{p o l}=0.5$ of $\partial E / \partial t$ is shown at a time step which corresponds to a maximum of the energy transfer. A phase space structure connected to the parallel transit resonance can be observed in Fig. (13-14) for both thermal and fast ions. The peak positions, i.e. the maxima of the energy exchange, occur at $v_{\|}$values predicted by Ref. 46 (black crosses in Fig. (13)). The full velocity space integrals of the $\partial E / \partial t$ structures shown in Fig. (13-14) correspond to the values of Fig. (12). The results of Fig. (13-14) have been obtained performing an average over four consecutive maxima of the total energy exchange.

\section{AUG EXPERIMENT BASED STUDIES}

In the following section a realistic ASDEX Upgrade experimental scenario with a strongly elongated plasma is investigated with linear electrostatic simulations studying the $n=0$ (EGAM) dynamics. This case refers to the AUG shot \#31213 at 0.841s [47], which contains significant fast ion effects and fast-ion driven modes. The major and minor radii are $R_{0}=1.62 \mathrm{~m}$ and $a=0.482 \mathrm{~m}$. The magnetic field on axis is 

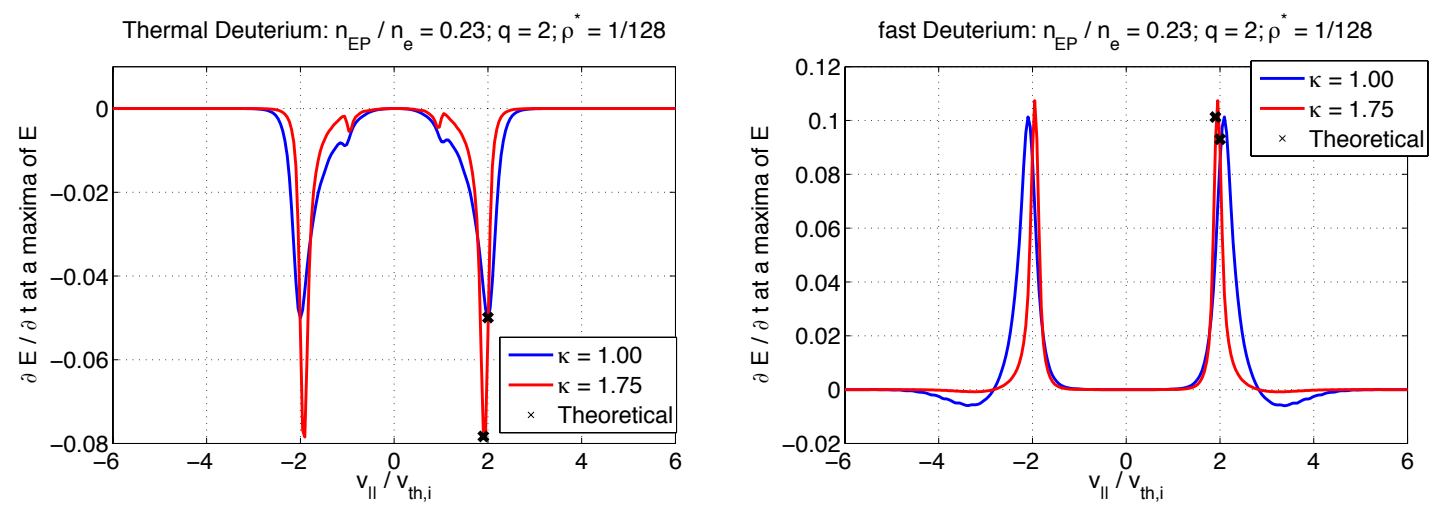

FIG. 13: Parallel velocity structure of $\partial E / \partial t$ for thermal (left) and fast (right) deuterium at $\mu=0$ for $\kappa=1.00$ (blue line) and $\kappa=1.75$ (red line) at fixed fast ion density $n_{f} / n_{e}=0.23$. The black crosses represent the different resonance positions for each value of the elongation, see Eq. (9).

$B_{0}=2.2 \mathrm{~T}$ and the safety factor has a reversed shear, with $q_{\min }\left(\rho_{p o l}=0.5\right)=2.3$. The plasma is composed by deuterium, electrons and is heated with $P_{\text {injected }}=2.5 \mathrm{MW}$ of deuterium-NBI. The tokamak magnetic geometry is provided with an experimental CHEASE equilibrium and is shown in Fig. (15) with the corresponding safety factor profile. The equilibrium is reconstructed from the experimental discharge via CLISTE and post-processed with CHEASE for interfacing to the gyrokinetic codes.

\section{A. Flat density and temperature profiles}

A first simplified setup removing any effects of the ion/electron temperature ratio, i.e with $T_{i}=T_{e}=1.603 \mathrm{keV}$, corresponding to $\rho^{*}=\rho_{s} / a=1 / 183.3$ and considering flat temperature and density profiles is considered in the following. The latter values have been taken from the full main-ion radial temperature profile at $\rho_{\text {pol }}=0.5$. 

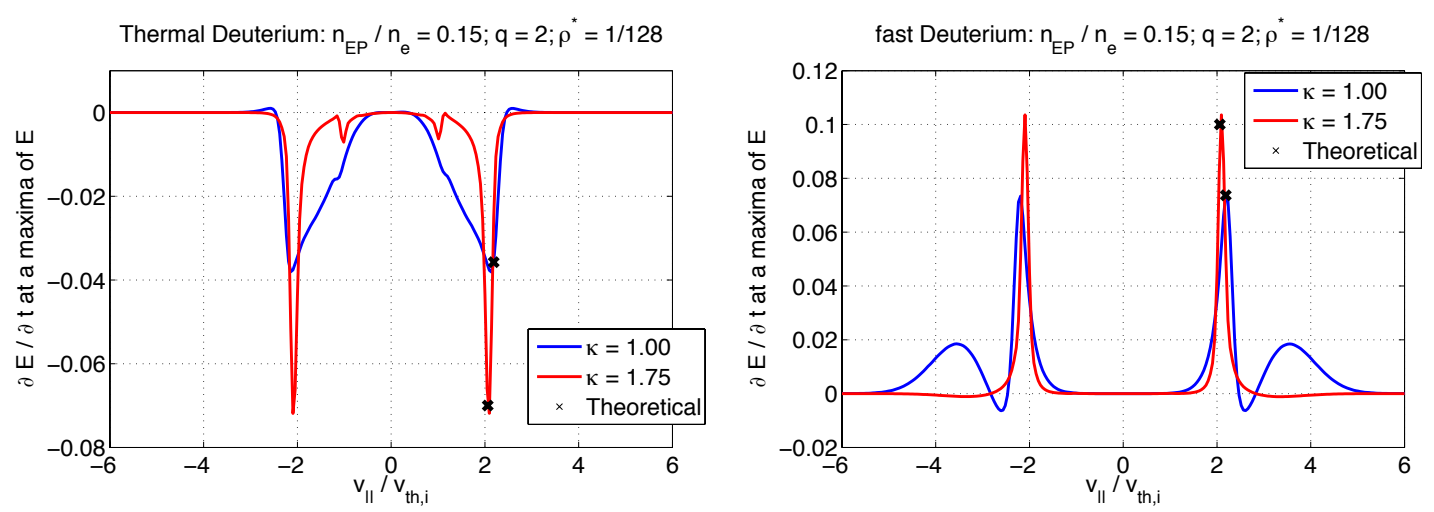

FIG. 14: Parallel velocity structure of $\partial E / \partial t$ at $\mu=0$ for the case $n_{f} / n_{e}=0.15$ and $\kappa=1.00$ (blue line), $\kappa=1.75$ (red line). The black crosses represent the different resonance positions identified with Eq. (9) for each value of the elongation.
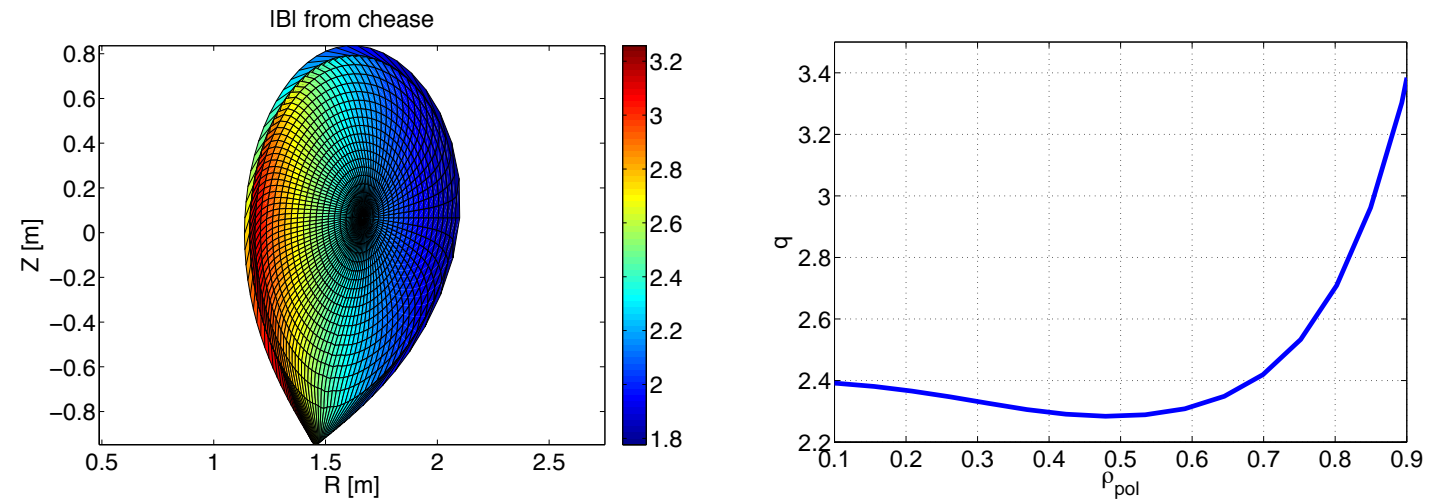

FIG. 15: a) Intensity of the equilibrium magnetic field on the flux-coordinate grid b) safety factor profile in the AUG discharge.

This first simplified analysis allows a reduction of the physical complexity of the system, removing any phase-mixing effects while keeping the effect of realistic plasma elongation. The electron density at this position is similarly determined to $n_{e}=$ $1.265 \cdot 10^{19} \mathrm{~m}^{-3}$. As before, fast ions are modelled with a bump-on-tail distribution 

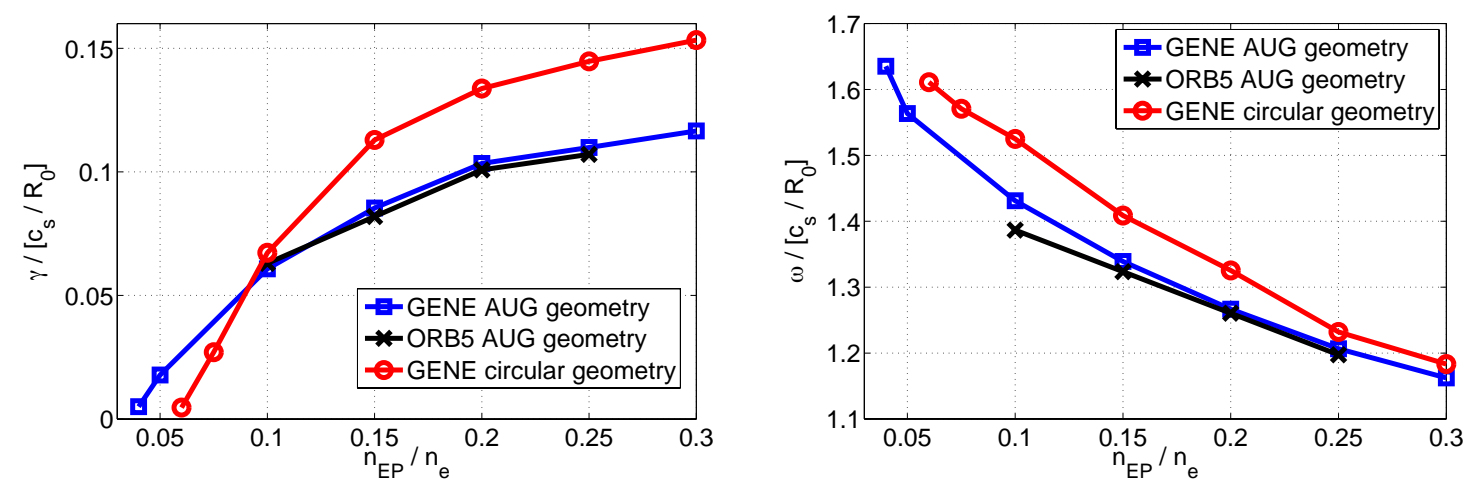

FIG. 16: Linear EGAM growth rates a) and frequencies b) are shown for different fast ions density concentration for the realistic CHEASE and a circular equilibrium for flat density and temperature profiles.

(Eq. (2)) - here with $\bar{v}_{\|}=2.83$ (= 4 in ORB5 units) in units of the thermal velocity and fast ion temperature set to the main ion one, i.e. $T_{E P}=T_{i}$. The considered radial simulation domain covers $0.15<\rho_{\text {pol }}<0.85$. For simplicity, electrons are considered adiabatic and an initial density perturbation of the form $n_{1}\left(\rho_{p o l}, t_{0}\right) \sim \sin \left(\pi \rho_{p o l}\right)$ is initialised with Dirichlet boundary conditions. In Fig. (16) the linear EGAM growth rates and frequencies obtained for the realistic CHEASE equilibria with GENE and ORB5 are shown and compared to the ones of a simplified circular geometry for different fast ions density concentrations. The safety factor profile of the circular geometry case is kept the same as the experimental one, see Fig. (15)b. The values of the growth rates and frequencies have been calculated at the radial position $\rho_{\text {pol }}=$ 0.25 , i.e. where the radial profile of the electrostatic potential is found to peak, as shown in Fig. (17). For the nominal fast ion density $n_{E P}=0.2 n_{e}$ at $\rho_{p o l}=0.5$, both codes agree well on the the values of the EGAM frequency and growth rate which are found to be $\omega=1.267 c_{s} / R_{0}=33.315 \mathrm{kHz}$ and $\gamma=0.103 c_{s} / R_{0}$ for the realistic AUG equilibrium. Fig. (16) furthermore demonstrates a pronounced effect of the magnetic 


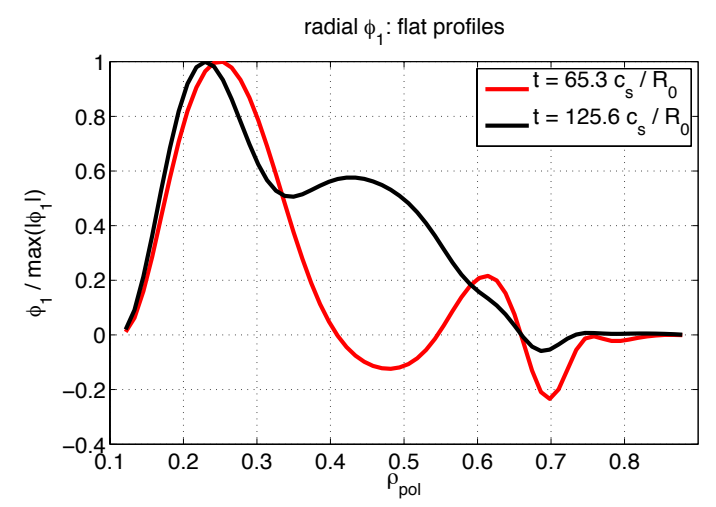

FIG. 17: Radial structure of the electrostatic potential at different simulation times for the case of flat density and temperature profiles in the AUG discharge.

geometry on the linear EGAM growth rates and frequencies. The plasma elongation $(\kappa)$ weakens the EGAM growth rates and slightly reduces the mode frequency. A change in the EGAM fast ion density threshold can be observed as well. Consistently with the results shown in the previous sections, the plasma elongation affects also the thermal damping, which unlike of the fast ion drive, strengthens with $\kappa$ and can became dominant for the cases of weak EGAM drive. The combined effect of the elongation on the thermal damping and fast ion drive is found to significantly affect the fast ion density threshold. However, it is worth specifying that close to the EGAM density threshold the effect of the kinetic electron damping might be not negligible anymore and should be taken into account [48]. Furthermore, the results shown in the previous sections were obtained in the case of strongly driven EGAMs. Close to the mode marginal stability the interaction between thermal damping and fast-ion drive might change significantly and affect the linear results. 

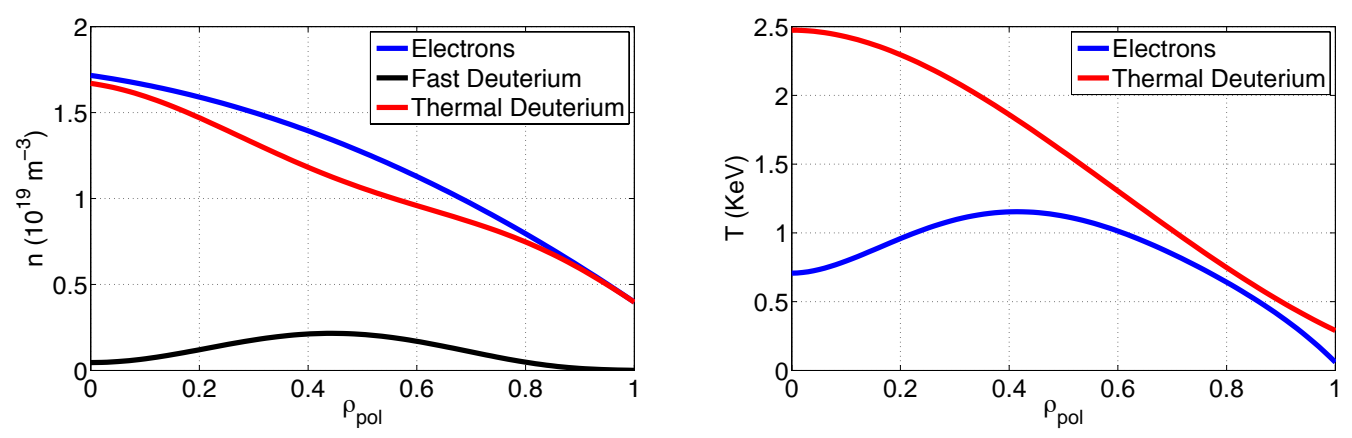

FIG. 18: Experimental a) density and b) temperature profiles. For the NBI fast deuterium a flat temperature profile is considered at the value $T_{E P}=30 \mathrm{keV}$. It is related to the injected energy.

\section{B. Realistic density and temperature profiles}

In the last section of this paper, the full realistic temperature and density profiles, shown in Fig. (18), are employed in the gyrokinetic analysis for each species. The fast ion population is modelled with the bump-on-tail distribution defined in Eq. (2), with a realistic temperature of $T_{E P}=30 \mathrm{keV}$. The latter choice also fixes $\bar{v}_{\| 1}$, i.e. the value of the shift of the bump-on-tail distribution function, which for the parameters employed in this section, is equal to $\bar{v}_{\|}=1.41$ ( $\sim 2$ in ORB5 units). The equivalent Maxwellian temperature imposes a constraint over the second order moment of the bump-on-tail distribution function to keep the same energy content. Furthermore, electrons are considered adiabatic and electrostatic simulations are performed with GENE with the radial domain covering $0.1<\rho_{\text {pol }}<0.9$. The logarithmic time trace of the perturbed electrostatic potential at $\rho_{\text {pol }}=0.5$ is shown in Fig. (19)a. By means of linear regression, the corresponding EGAM growth rate and frequency are determined to $\gamma=0.0465 c_{s} / R_{0}, \omega=2.013 c_{s} / R_{0}=44.69 \mathrm{kHz}$. Compared to values in the previous section, the EGAM growth rate for these realistic AUG based parameters 
appears to be relatively small. This finding is consistent with the analytic energy exchange formula, Eq. (10), which predicts a reciprocity between the mode/ion energy exchange term and the fast ion temperature. In the analysis at hand, the NBI fast Deuterium temperature has been fixed to a realistic value of $T_{E P}=30 \mathrm{keV}$, which is calculated from the NBI injected energy while thermal NBI Deuterium had been considered in the simplified setup in the previous section. The aforementioned increase in the fast ion temperature produces a net reduction of the energy exchange term between fast ions and the mode with a consequent reduction of the EGAM growth rate. Furthermore, we also observe a significant increase in the EGAM mode frequency. It is also possible to compare the experimentally measured frequency of the $n=0$ modes at $t=0.841 \mathrm{~s}$ and $\rho_{\text {pol }}=0.5$ with the values obtained by GENE with realistic density and temperature profiles. Despite the simplified setup here considered - adiabatic electrons, no nonlinear coupling and electrostatic simulations -, Fig. (19)b displays a surprisingly good agreement between the measurement of the EGAM frequency in the first linear phase and the simulation value. At later times, the EGAM mode enters a nonlinear chirping phase, which cannot be described with linear simulations. The particularly good agreement with the experimental frequency is achieved only by reasonably approximating modelling the fast ion temperature. For the case of $T_{E P}=T_{e}$, shown in the previous section, an underprediction of the mode frequency is observed. Moreover, a comparison with an ad-hoc circular equilibrium with a radial safety factor profile as in the realistic MHD equiblibrium reveals that the latter (with significant elongation) is also a crucial ingredient for achieving a good EGAM frequency agreement. The growth rate obtained with the circular equilibrium is found to increase of more than $50 \%$ up to $\gamma=0.0763 c_{s} / R_{0}$. The mode frequency, on the other hand, has a weaker dependence on the plasma elongation and it is found to increase by a factor of $\sim 15 \%$ up to $\omega=2.327 c_{s} / R_{0}$. In dimensional units, the mode frequency reads as $\omega=51.66 \mathrm{kHz}$, which is still qualitatively close 

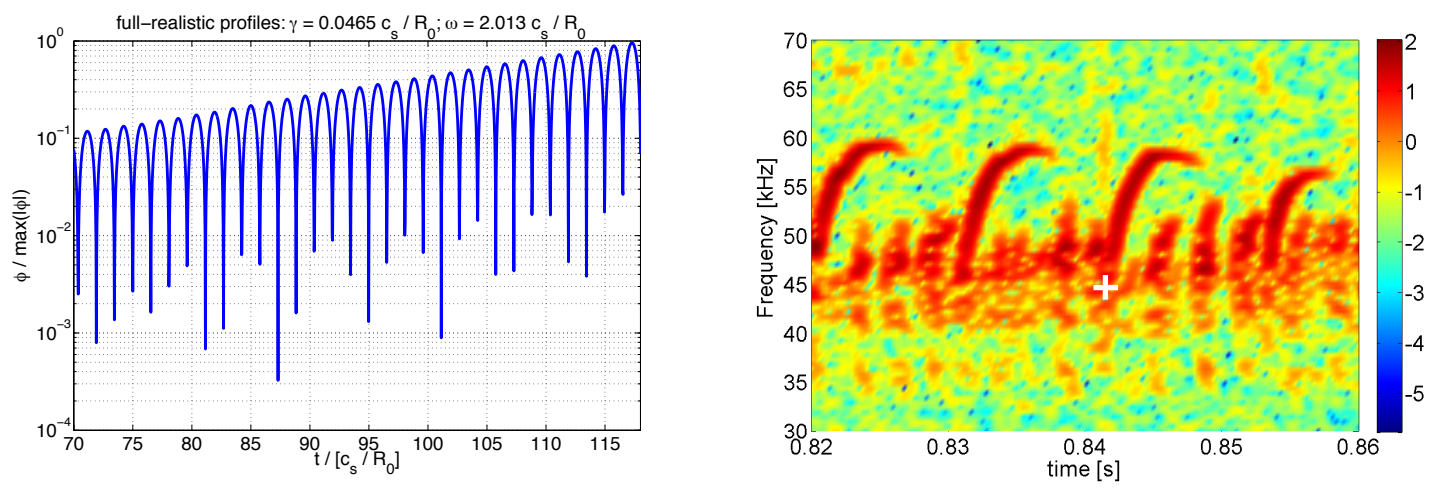

FIG. 19: a) Time trace of the perturbed electrostatic potential in logarithmic scale with the corresponding values of the EGAM growth rate and frequency for realistic density and temperature profiles; b) experimental time evolution of the frequency spectra of the AUG discharge \#31213. The white cross refers to the EGAM frequency obtained from GENE simulations.

to the experimental measurements. However, a quantitative agreement is achieved only by considering a more sophisticated model for the magnetic equilibrium which includes - amongst others - elongation effects. This finding is well in line with the dedicated analysis mentioned in Sec. V- VI. Furthermore, the radial structure of the electrostatic potential is also analysed at different simulation times, corresponding to maxima of $\phi_{1}$. As can be seen in Fig. (20), the mode is already localised around the maximum fast ion density at $\rho_{\text {pol }}=0.5$ at an early stage of the simulation. For different radial positions, the fast ion density decreases and the drive, which is already significantly weak at $\rho_{\text {pol }}=0.5$ barely overcomes the thermal damping and the mode is strongly weakened. However, for a more realistic comparison with the experimental measurements of the EGAM radial structure [28] - measured at $\rho_{\text {pol }} \sim 0.3$ -, a more accurate description of the fast ion background distribution function and of the nonlinear physics needs to be considered. 


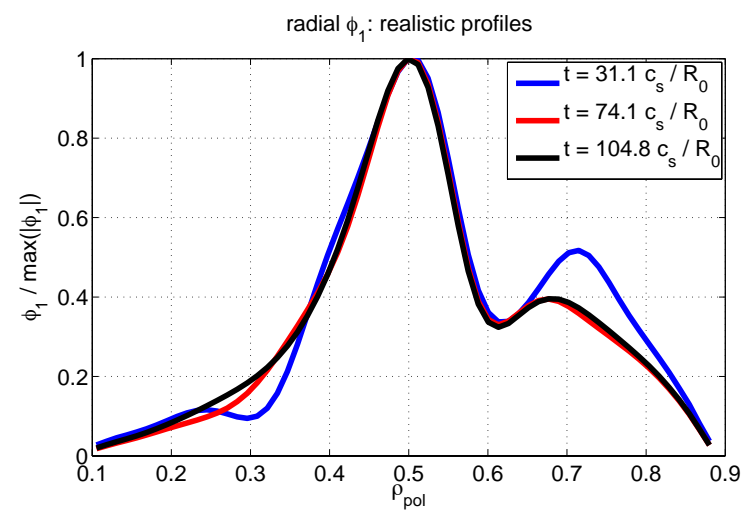

FIG. 20: Radial structure of the electrostatic potential at different simulation times for the case of realistic density and temperature profiles of the AUG discharge.

\section{CONCLUSIONS}

In the present paper, for the first time results obtained with the global version of the gyrokinetic code GENE which supports non-Maxwellian backgrounds are shown and benchmarked against the code ORB5. In particular, the effect of the plasma elongation on the EGAM dynamics is studied with both numerical simulations and reduced analytical models. To facilitate the comparison between theory and simulations, adiabatic electrons and electrostatic fluctuations are considered. Furthermore, a symmetric double bump distribution function is chosen for modelling the energetic ion population, while Maxwellian bulk ions are considered. It is found that the plasma elongation significantly weakens the EGAM growth rates, barely modifying the respective mode frequency. In particular, it introduces small order corrections on the parallel transit resonance position which affect the energy exchange between the fast ions and the mode, i.e. it modifies the phase space position in which the inverse Landau damping occurs. In particular, the resonant transit velocity decreases with the elongation, which, for the choice of parameters here considered, moves through 
region in phase space where the shape of the fast ion distribution is less favourable to exchange energy with the mode, with a consequent reduction of the linear growth rate. Finally, these finding have been applied to a real ASDEX Upgrade discharge with strongly elongated geometry. For this analysis realistic density and temperature profiles have been considered. Despite the approximations taken in our simulations regarding the EP distribution function and the adiabatic model for the electrons, a good agreement of the linear frequency obtained with numerical simulations and the onset of the EGAM frequency chirping cycle is found. Moreover, a significant effect of the plasma elongation on the EGAM instability threshold has been observed. The extension of the present numerical investigation to the nonlinear phase will be addressed in a dedicated publication.

\section{ACKNOWLEDGEMENT}

The simulations presented in this work were performed using the HYDRA cluster at the Rechenzentrum Garching (RZG), Germany. We acknowledge the CINECA award under the ISCRA initiative, for the availability of high performance computing resources and support. Furthermore, ORB5 simulations have been performed on the Marconi supercomputer within the framework of the OrbZONE and ORBFAST projects. This work has been carried out within the framework of the EUROfusion Consortium and has received funding from the Euratom research and training programme 2014- 2018 under grant agreement No 633053. The views and opinions expressed herein do not necessarily reflect those of the European Commission. The author would like to thank A. B. Navarro and F. Jenko for all the stimulating 
discussions, useful suggestions and comments.

[1] H. Doerk, A. Bock, A. Di Siena, E. Fable, T. Görler, F. Jenko, J. Stober, and The ASDEX Upgrade Team, Nucl. Fusion 58, 016044 (2018).

[2] P. Mantica, D. Strintzi, T. Tala, C. Giroud, T. Johnson, H. Leggate, E. Lerche, T. Loarer, A. G. Peeters, A. Salmi, S. Sharapov, D. Van Eester, P. C. de Vries, L. Zabeo, and K.-D. Zastrow, Phys. Rev. Lett. 102, 175002 (2009).

[3] P. Mantica, C. Angioni, C. Challis, G. Colyer, L. Frassinetti, N. Hawkes, T. Johnson, M. Tsalas, P. C. de Vries, J. Weiland, B. Baiocchi, M. N. A. Beurskens, A. C. A. Figueiredo, C. Giroud, J. Hobirk, E. Joffrin, E. Lerche, V. Naulin, A. G. Peeters, A. Salmi, C. Sozzi, D. Strintzi, G. Staebler, T. Tala, D. Van Eester, and T. Versloot, Phys. Rev. Lett. 107, 135004 (2011).

[4] C. L. Fiore, P. T. Bonoli, D. R. Ernst, M. J. Greenwald, E. S. Marmar, M. H. Redi, J. E. Rice, S. J. Wukitch, and K. Zhurovich, Plasma Phys. Control. Fusion 46, B281?B291 (2004).

[5] M. H. Redi, W. Dorland, C. L. Fiore, J. A. Baumgaertel, E. M. Belli, T. S. Hahm, G. W. Hammett, and G. Rewoldt, Phys. Plasmas 12, 072519 (2005).

[6] J. Citrin, F. Jenko, P. Mantica, D. Told, C. Bourdelle, J. Garcia, J. W. Haverkort, G. M. D. Hogeweij, T. Johnson, and M. J. Pueschel, Phys. Rev. Lett. 111, 155001 (2013).

[7] A. Di Siena, T. Görler, H. Doerk, E. Poli, and R. Bilato, Nucl. Fusion 58, 054002 (2018).

[8] N. Bonanomi, P. Mantica, A. Di Siena, E. Delabie, T. Johnson, E. Lerche, S. Menumir, M. Tsalas, D. Van Eester, and JET Contributors, Nucl. Fusion 58, 056025 (2018), 10.1088/1741-4326/aab733. 
[9] D. Zarzoso, Y. Sarazin, X. Garbet, R. Dumont, A. Strugarek, J. Abiteboul, T. CartierMichaud, G. Dif-Pradalier, V. Grandgirard, G. Latu, C. Passeron, and O. Thomine, Phys. Rev. Lett. 110, 125002 (2013).

[10] K. McGuire, R. Goldston, M. Bell, M. Bitter, K. Bol, K. Brau, D. Buchenauer, T. Crowley, S. Davis, F. Dylla, H. Eubank, H. Fishman, R. Fonck, B. Grek, R. Grimm, R. Hawryluk, H. Hsuan, R. Hulse, R. Izzo, R. Kaita, S. Kaye, H. Kugel, D. Johnson, J. Manickam, D. Manos, D. Mansfield, E. Mazzucato, R. McCann, D. McCune, D. Monticello, R. Motley, D. Mueller, K. Oasa, M. Okabayashi, K. Owens, M. Park, M. Reusch, N. Sauthoff, G. Schmidt, S. Sesnic, J. Strachan, C. Surko, R. Sluscher, H. Takahashi, F. Tenney, P. Thomas, H. Towner, J. Valley, and R. White, Phys. Rev. Lett. 51, 1925 (1983).

[11] H. L. Berk, C. J. Boswell, D. Borba, A. C. A. Figueiredo, T. Johnson, M. F. F. Nave, S. D. Pinches, S. E. Sharapov, and JET EFDA contributors, Nucl. Fusion 46, S888 (2006).

[12] P. Lauber, Phys. Rep. 533, 33 (2013).

[13] L. Chen and F. Zonca, Rev. Mod. Phys. 88, 015008 (2016).

[14] N. Winsor, L. Johnson, and M. Dawson, Phys. Plasmas 11, 2448 (1968).

[15] F. Zonca and L. Chen, Europhysics Lett. 83, 35001 (2008).

[16] M. N. Rosenbluth and F. L. Hinton, Phys. Rev. Lett. 80, 724 (1998).

[17] P. H. Diamond, S. I. Itoh, K. Itoh, and T. S. Hahm, Plasma Phys. Control. Fusion 47 (2005), 10.1088/0741-3335/47/5/R01.

[18] Z. Gao, Phys. Plasmas 20, 032501 (2013).

[19] Z. Qiu, I. Chavdarovski, A. Biancalani, and J. Cao, Phys. Plasmas 24, 072509 (2017).

[20] G. Y. Fu, Phys. Rev. Lett. 101, 185002 (2008).

[21] Z. Qiu, F. Zonca, and L. Chen, Plasma Phys. Control. Fusion 52, 095003 (2010).

[22] Z. Qiu, F. Zonca, and L. Chen, Plasma Science Tech. 12, 257 (2011). 
[23] D. Zarzoso, X. Garbet, Y. Sarazin, R. Dumont, and V. Grandgirard, Phys. Plasmas 19, 022102 (2012).

[24] J.-B. Girardo, D. Zarzoso, R. Dumont, X. Garbet, Y. Sarazin, and S. Sharapov, Phys. Plasmas 21, 092507 (2014).

[25] A. Biancalani, I. Chavdarovski, Z. Qiu, A. Bottino, D. Del Sarto, A. Ghizzo, Ö. Gürcan, P. Morel, and I. Novikau, J. Plasma Phys. 83, 725830602 (2017).

[26] R. Nazikian, G. Y. Fu, M. E. Austin, H. L. Berk, R. V. Budny, N. N. Gorelenkov, W. W. Heidbrink, C. T. Holcomb, G. J. Kramer, G. R. McKee, M. A. Makowski, W. M. Solomon, M. Shafer, E. J. Strait, and M. A. Van Zeeland, Phys. Rev. Lett. 101, 185001 (2008).

[27] T. Ido, M. Osakabe, A. Shimizu, T. Watari, M. Nishiura, K. Toi, k. Ogawa, K. Itoh, I. Yamada, R. Yasuhara, y. Yoshimura, and S. Kato, Nucl. Fusion 55, 083024 (2015).

[28] L. Horváth, G. Papp, P. Lauber, G. Por, A. Gude, V. Igochine, B. Geiger, M. Maraschek, L. Guimarais, V. Nikolaeva, G. I. Pokol, and The ASDEX Upgrade Team, Nucl. Fusion 56, 112003 (2016).

[29] X. T. Chen, W. Ding, L. M. Yu, X. Q. Ji, J. Q. Dong, Q. W. Yang, Y. Liu, L. W. Yan, Y. Zhou, W. Li, X. M. Song, S. Y. Chen, Z. B. Shi, and X. R. Duan, Phys. Lett. A 377, 387 (2013).

[30] A. Biancalani, A. Bottino, P. Lauber, and D. Zarzoso, Nucl. Fusion 54, 104004 (2014).

[31] D. Zarzoso, A. Biancalani, A. Bottino, P. Lauber, E. Poli, J.-B. Girardo, X. Garbet, and R. Dumont, Nucl. Fusion 54, 103006 (2014).

[32] H. Wang, Y. Todo, and C. Kim, Phys. Rev. Lett. 110, 155006 (2013).

[33] F. Jenko, W. Dorland, M. Kotschenreuther, and B. N. Rogers, Phys. Plasmas 7, 1904 (2000).

[34] S. Jolliet, A. Bottino, P. Angelino, R. Hatzky, T. M. Tran, B. F. Mcmillan, O. Sauter, K. Appert, Y. Idomura, and L. Villard, Comput. Phys. Comm. 177, 409 (2007). 
[35] A. Bottino and E. Sonnendrücker, J. Plasma Phys. 81, 435810501 (2015).

[36] T. Görler, X. Lapillonne, S. Brunner, T. Dannert, F. Jenko, F. Merz, and D. Told, Journal of Computational Physics 230, 7053 (2011).

[37] P. Xanthopoulos, H. E. Mynick, P. Helander, Y. Turkin, G. G. Plunk, F. Jenko, T. Görler, D. Told, T. Bird, and H. J. E. Proll, Phys. Rev. Lett. 113, 155001 (2014).

[38] A. Di Siena, T. Görler, H. Doerk, J. Citrin, T. Johnson, M. Schneider, E. Poli, and JET Contributors, J. Phys. Conf. Ser. 775, 012003 (2016).

[39] A. Di Siena, T. Görler, H. Doerk, R. Bilato, J. Citrin, T. Johnson, M. Schneider, E. Poli, and JET Contributors, Phys. Plasmas 25, 042304 (2018).

[40] X. Lapillonne, S. Brunner, T. Dannert, S. Jolliet, A. Marinoni, L. Villard, T. Görler, F. Jenko, and F. Merz, Phys. Plasmas 16, 032308 (2009).

[41] H. Lütjens, A. Bondeson, and O. Sauter, Comp. Phys. Comm. 97, 219 (1996).

[42] H. Sugama and T.-H. Watanabe, J. Plasma Phys. 74, 139 (2008).

[43] H. Sugama and T.-H. Watanabe, J. Plasma Phys. 72, 825 (2006).

[44] A. Bañón Navarro, P. Morel, M. Albrecht-Marc, D. Carati, F. Merz, T. Görler, and F. Jenko, Phys. Plasmas 18, 092303 (2011).

[45] P. Manas, Y. Camenen, S. Benkadda, W. A. Hornsby, and A. G. Peeters, Phys. Plasmas 22, 062302 (2015).

[46] Z. Gao, P. Wang, and H. Sanuki, Phys. Plasmas 15, 074502 (2008).

[47] P. Lauber, Off-axis NBI heated discharges at ASDEX Upgrade: EGAMs, RSAEs and TAE bursts. Presentation at the 13th Energetic Particle Physics TG Meeting, Padua, Italy 2014 https://portal.iter.org/departments/POP/ITPA/EP/EP/Documents/ 13/Presentation/01-01-Lauber.pdf.

[48] I. Novikau, A. Biancalani, A. Bottino, G. D. Conway, Ö. D. Gürcan, P. Manz, P. Morel, E. Poli, and A. Di Siena, Phys. Plasmas 24, 122117 (2017). 